\section{Extra attention for melanoma among elderly men}

\author{
Esther de Vries, Arjen Joosse and Jan W. Coebergh
}

We read with interest the Review article by Tsai and colleagues (Epidemiology and treatment of melanoma in elderly patients. Nat. Rev. Clin. Oncol. 7, 148-152; $\underline{2010})^{1}$ that offered a good overview on the issues regarding melanomas among the elderly. We would like to add a few comments, specifically concerning melanomas in elderly men, especially with respect to observations made in Europe. Compared with in the USA, differences in incidence and mortality according to age in European countries, such as The Netherlands, are less pronounced, and the difference in incidence between men and women of all ages is less dramatic (Figure 1). For example, in The Netherlands, the total number of newly diagnosed melanoma cases in 2007 was $3,722,58 \%$ of which were in patients aged $<60$ years at diagnosis. By contrast, the number of melanoma deaths in 2007 was $661,40 \%$ of which were in patients aged $<60$ years at diagnosis. ${ }^{2}$ As Tsai et al. ${ }^{1}$ numerous European countries the rates increased, ${ }^{3,4}$ especially among elderly men.

In addition to the relatively high in men persists at older age, and is growing primary tumors. ${ }^{9}$ mention, mortality rates in recent years had decreased in the USA, whereas in melanoma incidence and poor survival rates owing to their older age, elderly men with melanoma are also at greater risk of dying of melanoma than their female counterparts. Research indicates that the known melanoma survival disadvantage independent of prognostic factors such as age, body site, ulceration or tumor thickness, ${ }^{5-7}$ and even mitotic rate. ${ }^{8}$ The prognostic effect of age on survival might be largely dependent on the patient's gender. ${ }^{6}$ Interestingly, tumor behavior or tumor-host interaction may actually be different in elderly men compared with women, as these patients are more often than women diagnosed with rapid

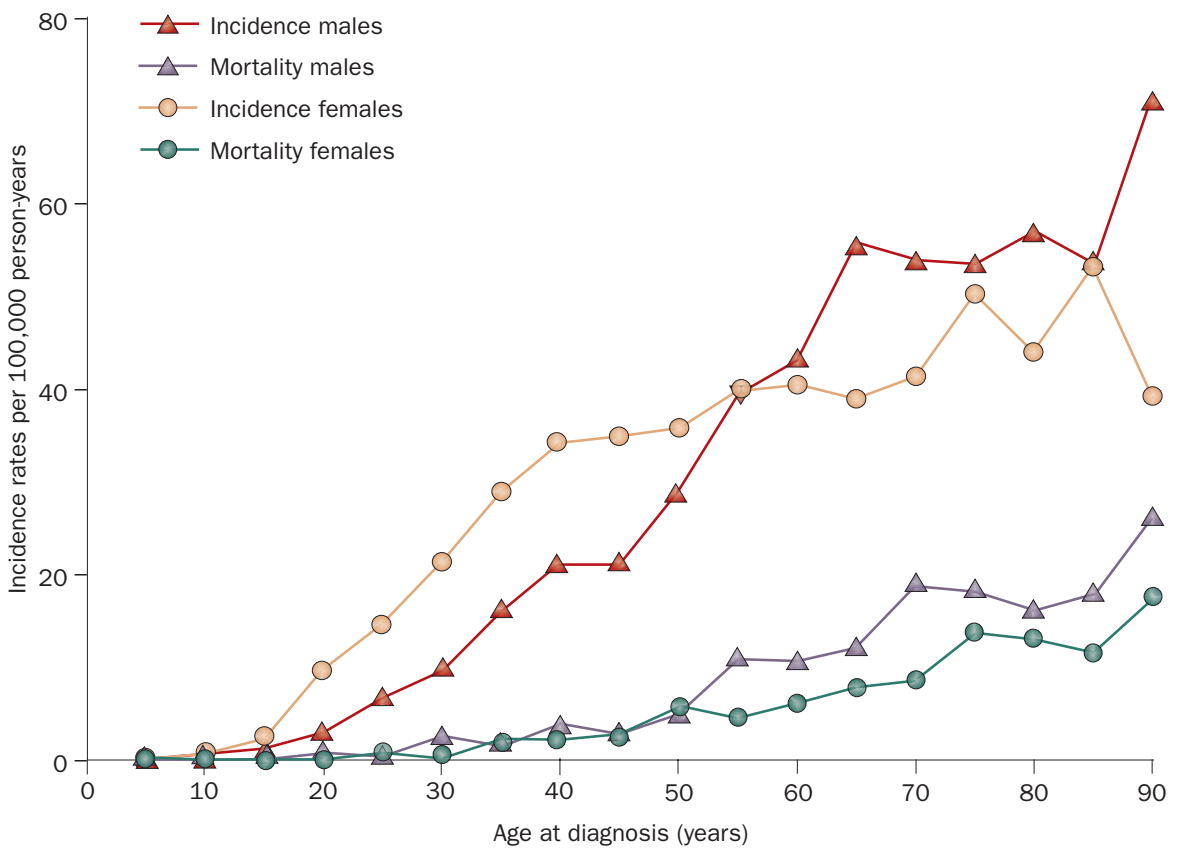

Figure 1 | Age-specific melanoma incidence and mortality in The Netherlands in 2007.
Summarizing the evidence, it is unlikely that delayed presentation or age alone can explain this phenomenon of poor survival in men with melanoma. Consequently, future screening campaigns should focus on men-especially older men. Unfortunately, most campaigns for early detection usually attract young women; for example, melanoma awareness, screening days or campaigns that aim to improve awareness on the risks of skin cancer and the need to inspect the skin for suspicious lesions in other ways. However, a study published in 2009 of the Belgian Euromelanoma campaign, in which the Belgian former Prime Minister featured prominently, showed that it is possible to attract elderly men to these campaigns. ${ }^{10}$

We feel that the public, physicians and researchers need to be aware of the substantial disadvantage in terms of melanoma survival in men at all ages. Elderly men deserve particular attention, as the combined disadvantages of being male, older and the relative high incidence of melanoma in this subgroup lead to high mortality rates. This heightened attention should be translated into more specifically targeted instructions to improve early detection among elderly men as well as to promote research into the mechanisms behind these survival disadvantages.

Department of Public Health, Erasmus MC University Medical Center, P. O. Box 2040, 3000 CA Rotterdam, The Netherlands (E. de Vries, A. Joosse, J. W. Coebergh).

Correspondence to: E. de Vries e.devries@erasmusmc.nl

doi:10.1038/nrclinonc.2010.1-c1

Competing interests

The authors declare no competing interests.

1. Tsai, S., Balch, C. \& Lange, J. Epidemiology and treatment of melanoma in elderly patients. Nat. Rev. Clin. Oncol. 7, 148-152 (2010).

2. Association of Comprehensive Cancer Centres [online], www.ikcnet.nl (2010).

3. de Vries, E. \& Coebergh, J. W. Melanoma incidence has risen in Europe. BMJ 331, 698 (2005).

4. La Vecchia, C. et al. Cancer mortality in Europe, 2000-2004, and an overview of trends since 1975. Ann. Oncol. doi:10.1093/annonc/mdp530.

5. de Vries, E. et al. Superior survival of females among 10,538 Dutch melanoma patients is independent of Breslow thickness, histologic 
type and tumor site. Ann. Oncol. 19, 583-589 (2008).

6. Lasithiotakis, K. et al. Age and gender are significant independent predictors of survival in primary cutaneous melanoma. Cancer 112, 1795-1804 (2008).

7. Balch, C. M. et al. Prognostic factors analysis of 17,600 melanoma patients: validation of the
American Joint Committee on Cancer melanoma staging system. J. Clin. Oncol. 19, 3622-3634 (2001).

8. Azzola, M. F. et al. Tumor mitotic rate is a more powerful prognostic indicator than ulceration in patients with primary cutaneous melanoma: an analysis of 3661 patients from a single center. Cancer 97, 1488-1498 (2003).
9. Liu, W. et al. Rate of growth in melanomas: characteristics and associations of rapidly growing melanomas. Arch. Dermatol. 142, 1551-1558 (2006).

10. Del Marmol, V. et al. A Prime Minister managed to attract elderly men in a Belgian Euromelanoma campaign. Eur J. Cancer 45, 1532-1534 (2009). 